SUNY-NTG-94-37

\title{
Heavy Mesons in a Random Instanton Gas [
}

\author{
S. Chernyshev, M. A. Nowak and I. Zahed \\ Department of Physics \\ SUNY at Stony Brook \\ Stony Brook, New York 11794
}

\begin{abstract}
We analyze the correlation function of a meson with one heavy and one light quark in inverse powers of the heavy quark mass $m_{Q}$ using a succession of Foldy-Wouthuysen-type transformations prior to radiative corrections. We evaluate the correlator to order $m_{Q}^{0}$ in a random and dilute gas of instantons, using the planar approximation. We show, in leading order in the density, that the heavy quark mass is shifted to the order $m_{Q}^{0}$ and that the induced interaction between the heavy and light quarks is attractive. We also find it to be an order of magnitude smaller than the 't Hooft interaction between two light quarks. The shift in the heavy quark mass is related to the perimeter law of large Wilson loops. The relevance of these results for general hadronic correlators with heavy quarks is discussed.
\end{abstract}

SUNY-NTG-94-37

July 1994

*Supported by the Department of Energy under Grant No. DE-FG02-88ER40388. 
The instanton model for the QCD ground state [1] offers an interesting framework for the discussion of soft physics from first principles. While instantons do not confine color in pure Yang-Mills theory, they provide a simple mechanism for the spontaneous breaking of chiral symmetry in QCD. Extensive calculations using instantons both analytically and numerically suggest that the instantons in the vacuum form a dilute gas [2]. This may be understood by noting that the instanton-antiinstanton interaction is not dipole like at large distances but screened by the fermions over distances on the order of $1 / 2 \mathrm{fm}$. A dilute system of instantons behaves as a free gas. As a result, the singlet $\eta^{\prime}$ is heavy and the topological susceptibility is small.

Hadrons with a heavy quark exhibit a new type of symmetry: invariance under spin flip of the heavy quark. This symmetry has been used in combination with chiral symmetry (Heavy Quark Effective Theory) [3] to understand the structure and decay properties of heavy-light systems. The main observation is that if the mass of one quark is taken to infinity and factorized, the physics of the remaining system is soft and constrained by chiral dynamics. The analysis of the soft physics in heavy-light systems can be assessed quantitatively using QCD lattice simulations, or qualitatively using QCD inspired models.

In this letter, we investigate the effects of a dilute gas of instantons on mesons composed of one heavy and one light quark. We show that the heavy meson correlator may be systematically investigated in inverse powers of the heavy quark mass using standard Foldy-Wouthuysen transformations [4] and before the inclusion of radiative corrections. We analyze the heavy meson correlator in the planar approximation and for a dilute gas of instantons. To leading order in the density, the instantons shift the heavy quark mass as first noted by Diakonov, Petrov and Pobylitsa [5], and induce an attractive scalar-color interaction between the heavy and light quarks that is an order of magnitude weaker than 
the induced 't Hooft interaction between two light quarks [6]. The shift in the heavy quark mass is related to the perimeter law of the Wilson loop and reminiscent of the classical Coulomb energy. The instanton gas screens but does not confine. Our construction is immediately amenable to heavy baryons. The instanton size $\rho$ is fixed to $1 / 3 \mathrm{fm}$ throughout and the instanton density $n_{*}$ is fixed to $1 \mathrm{fm}^{-4}$.

1. Consider the following meson correlator in Minkowski space

$$
\mathcal{C}_{\Gamma}\left(x, x^{\prime}\right)=\left\langle 0\left|T\left(\bar{q} \Gamma \psi(x) \bar{\psi} \Gamma q\left(x^{\prime}\right)\right)\right| 0\right\rangle
$$

with $\Gamma=(\mathbf{1}, \gamma) \times(\mathbf{1}, T)$ an arbitrary spin-flavor channel. In the regime where the mass $m_{Q}$ of the field $\psi$ is heavy, the correlator (1) may be analysed systematically in $1 / m_{Q}$ by redefining the field $\psi$ using the Foldy-Wouthuysen (FW) construction [7]. In the bare version and to leading order in $1 / m_{Q}$, the transformation reads

$$
\psi(x) \sim e^{-i \gamma_{0} m_{Q} t} e^{-i \sigma_{0 i}\left[\nabla^{0}, \nabla^{i}\right] / 4 m_{Q}^{2}} e^{-i \vec{\gamma} \cdot \vec{\nabla} / 2 m_{Q}} Q(x)
$$

where $\nabla=\partial-i A$ and with $g$ (the gauge coupling) set to one. The first transformation rescales the momenta, the second eliminates the odd parts, and the third removes the mass term. The successive transformations in (2) are vector-like, unitary and gauge-covariant. Thus, they are norm-preserving and anomaly-free. In terms of (2) the QCD part of the action for the heavy field $\psi$ becomes

$$
\mathcal{L}_{\psi} \sim \bar{Q} i \gamma^{0} \nabla^{0} Q+\bar{Q}\left(-\frac{\vec{\nabla}^{2}}{2 m_{Q}}-\frac{\vec{\sigma} \cdot \vec{B}}{2 m_{Q}}\right) Q
$$

with $B^{i}=-i \epsilon^{i j k}\left[\nabla^{i}, \nabla^{j}\right]$. Equation (3) has the expected FW form to order $m_{Q}^{-1}$. Under (2) the heavy meson source shifts

$$
\bar{q} \Gamma \psi \sim \bar{q} \Gamma e^{-i \gamma^{0} m_{Q} t}\left(1-\frac{i \vec{\gamma} \cdot \vec{\nabla}}{2 m_{Q}}\right) Q
$$


As a result, the correlator (11) takes the generic form

$$
\mathcal{C}_{\Gamma} \sim\left\langle 0\left|\bar{q} \Gamma e^{-i \gamma^{0} m_{Q}\left(t-t^{\prime}\right)} Q \bar{Q} \Gamma q\right| 0\right\rangle+\left\langle 0\left|\bar{q} \Gamma e^{-i \gamma^{0} m_{Q}\left(t+t^{\prime}\right)}\left[Q \bar{Q}, \frac{i \vec{\gamma} \cdot \overleftarrow{\nabla}}{2 m_{Q}}\right] \Gamma q\right| 0\right\rangle
$$

This construction can be extended to arbitrary orders in $1 / m_{Q}$. Since the FW transformation preserves manifest gauge invariance order by order in the heavy quark mass, the issue of renormalizability holds order by order in $1 / m_{Q}$. Our construction refers to the bare quark mass $m_{Q}$. We are assuming that the expansion is not upset by renormalization.

We note that the second term in (5) induces mixing between the particle and the antiparticle content of the correlator. This mixing follows from the redefinition of the sources and not the action. As we are interested in the case where $m_{Q}$ is large ( nonrelativistic limit ) it is convenient to take the projected sources $\Gamma_{ \pm}=\Gamma\left(1 \pm \gamma^{0}\right) / 2$ instead of the unprojected sources $\Gamma$. Since $\Gamma_{ \pm} \vec{\gamma} \cdot \vec{\nabla} \Gamma_{ \pm}=0$ the mixing part in (可) drops. Thus, in Euclidean space

$$
\mathcal{C}_{\Gamma}^{ \pm}\left(x, x^{\prime}\right) \sim-e^{\mp m_{Q}\left(\tau-\tau^{\prime}\right)}\left\langle 0\left|\operatorname{Tr}\left(\Gamma_{ \pm} S_{Q}\left(x, x^{\prime}\right) \Gamma_{ \pm} S\left(x^{\prime}, x\right)\right)\right| 0\right\rangle
$$

where $S$ is the propagator of the light quark, and $S_{Q}$ is the heavy quark propagator,

$$
S_{Q} \sim S_{\infty}+S_{\infty}\left(-\frac{\vec{\nabla}^{2}}{2 m_{Q}}-\frac{\vec{\sigma} \cdot \vec{B}}{2 m_{Q}}\right) S_{\infty}+O\left(m_{Q}^{-2}\right)
$$

with $S_{\infty}=\gamma_{4} / i \nabla_{4}$ being the free part. The order $m_{Q}^{0}$ has been considered by many authors [3]. Terms of order $m_{Q}^{-2}$ and higher can be sought along the same lines.

The present construction has the advantage of transforming the complete correlator and action into an effective correlator and an effective action with manifest power counting in $1 / m_{Q}$. The transformation does not rely on the commonly used momentum decomposition $p_{Q}=m_{Q} v+k$, and as such does not suffer from the ambiguities associated with the redefinition of the heavy quark velocity $v$ [8]. We have explicitly used the rest frame 
of the heavy quark, thus $v=(1, \overrightarrow{0})$ in our case. The derivation can be easily generalized to arbitrary frames.

2. We now proceed to evaluate (7) to order $m_{Q}^{0}$ in a random gas of instantons. In general, the correlator $\mathcal{C} \sim\left\langle S \otimes S_{\infty}\right\rangle$ receives contributions from both planar and nonplanar graphs and thus is difficult to analyze. However, to leading order in $1 / N_{c}$ the planar graphs dominate [5, 10]. To this order, the $\eta^{\prime}$ is massive (even in the chiral limit) and the instantons are screened. A dilute system of instantons and antiinstantons with zero net topological charge behaves as a free gas. The system screens but does not confine (see below). This notwithstanding, the planar graphs may be resummed using a Bethe-Salpeter-type equation. Following Pobylitsa [10], the inverse correlator (6) after resummation reads

$$
\mathcal{C}^{-1} \sim S^{-1} \otimes S_{\infty}^{T-1}-\sum_{I, \bar{I}}\left\langle\left(S-\not_{I}^{-1}\right)^{-1} \otimes\left(S_{\infty}-\not_{4, I}^{-1}\right)^{T-1}\right\rangle
$$

The upper script $T$ is short for transpose. In the planar approximation, the light propagator satisfies the integral equation

$$
S^{-1}=S_{0}^{-1}-\sum_{I, \bar{I}}\left\langle\left(A_{I}^{-1}-S\right)^{-1}\right\rangle
$$

For massless quarks $S_{0}^{-1}=+i \not \partial$, while the infinitely heavy quark propagator satisfies

$$
S_{\infty}^{-1}=S_{*}^{-1}-\sum_{I, \bar{I}}\left\langle\left(A_{4, I}^{-1}-S_{\infty}\right)^{-1}\right\rangle
$$

where $A_{4}=\gamma^{4} A^{4}$ and $S_{*}=i \gamma^{4} \partial^{4}$. The sum is over all instantons and antiinstantons and the averaging is over their position $z_{I}$ and color orientation $U_{I}$. Generically

$$
\sum_{I, \bar{I}} \rightarrow \frac{N}{2}\left(\frac{1}{V_{4}} \int d^{4} z_{I}\right) \int d U_{I}+(I \rightarrow \bar{I}) \sim n \int d^{4} z(I+\bar{I})
$$

where $n=N / 2 V_{4} N_{c}=n_{*} / 2 N_{c}$. The last substitution follows from color averaging over two $U_{I}$ 's for illustration. The diluteness factor of the gas is given by the dimensionless combination $n \rho^{4} \sim 10^{-3}$, with $n \sim N_{c}^{0}$ since $n_{*} \sim N_{c}$ [2]. 
In the dilute gas approximation, the nested integral equations (8-10) may be iterated in $n$. To leading order

$$
\mathcal{C}^{-1} \sim S^{-1} \otimes S_{\infty}^{T,-1}-n \int d^{4} z \operatorname{Tr}_{C}\left([L]_{I} \otimes[H]_{I}+I \rightarrow \bar{I}\right)
$$

with

$$
[L]_{I} \otimes[H]_{I}=\left[S_{0}^{-1}\left(\frac{\left|\Phi_{0}\right\rangle\left\langle\Phi_{0}\right|}{i \sqrt{n} \Sigma_{0}}-S_{0}\right) S_{0}^{-1}\right] \otimes\left[S_{*}^{-1}\left(\frac{1}{i \gamma^{4} \nabla_{4 I}}-S_{*}\right) S_{*}^{-1}\right]^{T}
$$

Here $\left|\Phi_{0}\right\rangle$ is the normalized fermionic zero mode in the one-instanton background, and $\Sigma_{0}=\left\langle\Phi_{0}|\Sigma| \Phi_{0}\right\rangle \sim(240 \mathrm{MeV})^{-1}$. We note that the effects of the planar resummation is to generate an effective mass for the zero mode in (13). This result is directly related to the induced constituent quark mass. Indeed, resumming the planar graph for the single quark propagator shows that $S^{-1} \sim S_{0}^{-1}+i \sqrt{n} \Sigma$, where $\Sigma$ is a space-spin-dependent kernel that satisfies the following gap-like equation [10]

$$
\Sigma=\int d^{4} z \operatorname{Tr}_{C}\left(S_{0}^{-1} \frac{\left|\Phi_{0}><\Phi_{0}\right|}{\Sigma_{0}} S_{0}^{-1}+I \rightarrow \bar{I}\right)
$$

with $\Sigma_{0}$ following from (14) by taking the expectation value in the zero mode state. The shift in the light quark mass is

$$
\Delta M_{q} \sim \sqrt{n}<x_{-\infty}|\Sigma| x_{+\infty}>\sim \sqrt{n}\left(\frac{4 \pi^{2} \rho^{2}}{\Sigma_{0}}\right) \sim 420 \mathrm{MeV}
$$

Similarly for the heavy quark propagator, we have $S_{\infty}^{-1} \sim S_{*}^{-1}+i n \Theta$, where $\Theta$ is a t-dependent kernel satisfying

$$
\Theta=\int d^{4} z \operatorname{Tr}_{C}\left(S_{*}^{-1}\left(\frac{1}{i \gamma^{4} \nabla_{4, I}}-S_{*}\right) S_{*}^{-1}+I \rightarrow \bar{I}\right)
$$

In coordinate space, the heavy quark propagator in the one instanton background reads

$$
<x\left|\frac{1}{i \nabla_{4, I}}\right| 0>=\delta(\vec{x}) \theta(\tau) \mathbf{P} e^{i \int_{0}^{\tau} d s A_{4}\left(x_{s}-z_{I}\right)}
$$


with $x_{s}=(s, \vec{x})$. Inserting (17) into $(\sqrt{16})$ and using the one-instanton configuration

$$
A_{\mu}^{a}(x)=+\bar{\eta}_{\mu \nu}^{a} x_{\nu}\left(\frac{1}{x^{2}}-\frac{1}{x^{2}+\rho^{2}}\right)
$$

yields for large times

$$
<x_{-\infty}|\Theta| x_{+\infty}>\sim\left(-32 \pi \rho^{3} \gamma^{4}\right) \int_{0}^{\infty}\left(x \cos \left(\frac{\pi x}{2 \sqrt{1+x^{2}}}\right)\right)^{2}=\left(-32 \pi \rho^{3} \gamma^{4} I_{Q}\right)
$$

The corresponding shift in the heavy quark mass follows from the effective vertex (16)

$$
\Delta M_{Q}=32 \pi n \rho^{3} I_{Q} \sim 16 \pi n \rho^{3} \sim 70 \mathrm{MeV}
$$

as first suggested by Diakonov, Petrov and Pobylitsa [5]. Note that the shift in the heavy quark mass is of order $n$ as opposed to the shift in the light quark mass which is of order $\sqrt{n}$, almost an order of magnitude smaller. This is simply the classical Coulomb energy of a heavy quark. A similar result has been recently obtained by Bigi, Shifman, Uraltsev and Vainshtein and also S. Narison [11]. Remarkably, their estimate using the infrared renormalon suggests $\Delta M_{Q} \sim 50 \mathrm{MeV}$. and $\Delta M_{Q} \sim 70 \mathrm{MeV}$ respectively.

3. The shift in the heavy quark mass to order $m_{Q}^{0}$ is related to the behavior of large Wilson loops in the random instanton gas. Indeed, the Wilson loop can be rewritten as follows

$$
\left\langle\operatorname{Tr}_{c} \mathbf{P} e^{i \int_{T \times L} d x \cdot A}\right\rangle=-\left\langle\operatorname{Tr}_{c}\left(\mathbf{P} e^{i \int_{T_{1}} d x \cdot A\left(T_{1}\right)} \mathbf{P} e^{i \int_{L_{1}} d x \cdot A\left(L_{1}\right)} \mathbf{P} e^{i \int_{T_{2}} d x \cdot A\left(T_{2}\right)} \mathbf{P} e^{i \int_{L_{2}} d x \cdot A\left(L_{2}\right)}\right)\right\rangle
$$

where $T_{1,2}$ and $L_{1,2}$ are the paths shown in Fig. 1. $A(T, L)=\sum_{I} A_{I}(T, L)$ is the sum of the instanton and antiinstanton gauge configurations projected onto the lines $T, L$. For large Wilson loops $T, L>>1 / \rho$, the heavy quarks (line integrals) no longer interact with each other. As a result, (21) factorizes into the trace of the product of four "heavy quark" propagators (Wilson lines), each averaged over the ensemble of instantons and 
antiinstantons, and evaluated for large separations $T$ and $L$ respectively. The above discussion shows that the heavy quark propagator acquires a mass $\Delta M_{Q}$ asymptotically. Thus

$$
<\operatorname{Tr}_{c} \mathbf{P} e^{i \int_{T \times L} d x \cdot A}>\sim-e^{-\Delta M_{Q}(2 T+2 L)}
$$

Large Wilson loops obey a perimeter law with $\sigma_{P}=\Delta M_{Q}$. By restricting the discussion to classical fields the nasty issue of the divergences in the Coulomb energy does not arise. It would be interesting to find out, how (22) compares with unquenched lattice calculations before and after cooling. A similar behavior is also present in the Schwinger model.

In $\mathrm{QCD}$, large Wilson loops are expected to obey a perimeter law. Does this mean that dilute instanton systems reflect faithfully on unquenched QCD ? We do not think so. In the presence of light quarks, the instantons and antiinstantons in the vacuum are screened over distance scales on the order of $1 / 2 \mathrm{fm}$. The screening is due to feedback of the quarks on the instanton configurations. While the effect is naively down by $1 / N_{c}$, since the instanton density grows with $N_{c}$, the screening persists to order $N_{c}^{0}$. This screening causes the topological susceptibility to be small and the $\eta^{\prime}$ to be massive. Dilute instanton systems in the presence of light quarks behave as a free gas. The operating mechanism is screening and not confinement. The lack of confinement is dramatically seen in (8) where the first term in the inverse correlator reflects on a dressed heavy-light quark cut. This problem occurs in all correlators.

4. The above notwithstanding, the inverse correlator (13) allows for an immediate interpretation in terms of effective interactions. In the long wavelength limit, the instanton size is small, and a local effective interaction between the effective fields $\mathbf{Q}$ and $\mathbf{q}$ (as opposed to the "fundamental" fields $Q$ and $q$ ) can be derived much like the 't Hooft interaction between the light effective fields $\mathbf{q}$. To show this, let $(x, y)$ and $\left(x^{\prime}, y^{\prime}\right)$ be 
the coordinates of the light and heavy quarks respectively before and after they have interacted with an instanton (antiinstanton). From Bethe-Salpeter equation we read the vertex

$$
\Gamma\left(x, y, x^{\prime}, y^{\prime}\right)=-i n N_{c} \int d^{4} z \int d U\left(\left\langle x|[L]| x^{\prime}\right\rangle \otimes\left\langle y|[H]| y^{\prime}\right\rangle+I \rightarrow \bar{I}\right)
$$

This vertex function gives rise to an effective action $\mathcal{S}_{I}$. Averaging over the instanton orientation and position yields in the long wavelength (local) approximation

$$
\mathcal{L}_{q Q}=n\left(-\frac{16 \pi \rho^{3} I_{Q}}{N_{c}}\right)\left(\frac{4 \pi^{2} \rho^{2}}{\sqrt{n} \Sigma_{0}}\right)\left(i \mathbf{Q}^{\dagger} \frac{1+i \gamma_{4}}{2} \mathbf{Q} i \mathbf{q}^{\dagger} \mathbf{q}+\frac{1}{4} i \mathbf{Q}^{\dagger} \frac{1+i \gamma_{4}}{2} \lambda^{a} \mathbf{Q} i \mathbf{q}^{\dagger} \lambda^{a} \mathbf{q}\right)
$$

The first bracket in (24) arises from the heavy quark part and the second bracket from the light quark part. Wick-rotating to Minkowski space gives

$$
\mathcal{L}_{q Q}=-\left(\frac{\Delta M_{Q} \Delta M_{q}}{2 n N_{c}}\right)\left(\overline{\mathbf{Q}} \frac{1+\gamma^{0}}{2} \mathbf{Q} \overline{\mathbf{q}} \mathbf{q}+\frac{1}{4} \overline{\mathbf{Q}} \frac{1+\gamma^{0}}{2} \lambda^{a} \mathbf{Q} \overline{\mathbf{q}} \lambda^{a} \mathbf{q}\right)
$$

which is to be compared with the 't Hooft vertex for two light flavors $\mathbf{q}=(\mathbf{u}, \mathbf{d})$

$$
\mathcal{L}_{q q}=\left(\frac{\Delta M_{q}^{2}}{n N_{c}}\right)\left(\operatorname{det} \overline{\mathbf{q}}_{R} \mathbf{q}_{L}+\operatorname{det} \overline{\mathbf{q}}_{L} \mathbf{q}_{R}\right)
$$

The ratio of the strengths is $\kappa_{q Q} / \kappa_{q q}=\Delta M_{Q} / 2 \Delta M_{q} \sim 0.08$ and of order $N_{c}^{0}$, with $\kappa_{q Q} \sim \sqrt{n} / N_{c}$

Similar arguments may be applied to $\bar{Q} Q$ mesons as well. The induced effective Lagrangian for heavy-light and heavy-heavy mesons is given by

$$
\begin{aligned}
\mathcal{L} & =\overline{\mathbf{q}}\left(i \not \partial-\Delta M_{q}\right) \mathbf{q}+\overline{\mathbf{Q}} \frac{1+\gamma^{0}}{2}\left(i \partial_{t}-\Delta M_{Q}\right) \mathbf{Q} \\
& -\kappa_{q Q}\left(\overline{\mathbf{Q}} \frac{1+\gamma^{0}}{2} \mathbf{Q} \overline{\mathbf{q}} \mathbf{q}+\frac{1}{4} \overline{\mathbf{Q}} \frac{1+\gamma^{0}}{2} \lambda^{a} \mathbf{Q} \overline{\mathbf{q}} \lambda^{a} \mathbf{q}\right) \\
& -\kappa_{Q Q}\left(\overline{\mathbf{Q}} \frac{1+\gamma^{0}}{2} \mathbf{Q} \overline{\mathbf{Q}} \frac{1+\gamma^{0}}{2} \mathbf{Q}+\frac{1}{4} \overline{\mathbf{Q}} \frac{1+\gamma^{0}}{2} \lambda^{a} \mathbf{Q} \overline{\mathbf{Q}} \frac{1+\gamma^{0}}{2} \lambda^{a} \mathbf{Q}\right)
\end{aligned}
$$


where $\kappa_{Q Q}=\Delta M_{Q}^{2} / 2 n N_{c}$. For heavy baryons the construction applies as well. For $q q Q$ systems the answer in Minkowski space reads

$$
\begin{aligned}
& \mathcal{L}_{q q Q}=-\left(\frac{\Delta M_{Q} \Delta M_{q}^{2}}{2 n^{2} N_{c}^{2}}\right)\left(\overline{\mathbf{Q}} \frac{1+\gamma^{0}}{2} \mathbf{Q}\left(\operatorname{det} \overline{\mathbf{q}}_{L} \mathbf{q}_{R}+\operatorname{det} \overline{\mathbf{q}}_{R} \mathbf{q}_{L}\right)+\right. \\
& \left.\frac{1}{4} \overline{\mathbf{Q}} \frac{1+\gamma^{0}}{2} \lambda^{a} \mathbf{Q}\left(\operatorname{det} \overline{\mathbf{q}}_{L} \lambda^{a} \mathbf{q}_{R}+\operatorname{det} \overline{\mathbf{q}}_{R} \lambda^{a} \mathbf{q}_{L}\right)\right)
\end{aligned}
$$

We note that $\kappa_{q q Q} \sim n^{0} / N_{c}^{2}$. A comparison with the conventional 't Hooft determinantal interaction shows that $\kappa_{q q Q} / \kappa_{q q q}=\kappa_{q Q} / \kappa_{q q} \sim 0.08$. The ratio of the strengths in heavy to light baryons is the same as the ratio of the strengths in heavy to light mesons. We note that both (27) and (28) are invariant under a spin flip of the heavy quark $S U(2)_{Q}$ and $U_{A}(1)$ violating, and that (28) is chiral $S U(2)_{L} \times S U(2)_{R}$ invariant.

In terms of the effective quark fields $\mathbf{Q}$ and $\mathbf{q}$ the dynamics is the one of the constituent quark model to order $m_{Q}^{0}$. For heavy light mesons, the spectrum is

$$
M_{q Q}=\left(m_{Q}+\Delta M_{Q}\right)+\left(m_{q}+\Delta M_{q}\right)+\alpha_{q Q} \kappa_{q Q}\left(\mathbf{1}+\frac{1}{4} \lambda_{q}^{a} \cdot \lambda_{Q}^{a}\right)
$$

with $\alpha_{q Q}=\left|\phi_{q Q}(0)\right|^{2} \sim 1 / a_{q Q}^{3}$, where $\phi_{q Q}(0)$ is the $q Q$-wavefunction at the origin and $a_{q Q}$ its typical size. Using the Van-Royen-Weisskopf construction [12], we obtain

$$
a_{q Q}^{2} \sim \frac{N_{c}}{2 \pi^{2} f_{q Q}^{2}}
$$

with $a_{q Q} \sim 0.26 \mathrm{fm}$ for $f_{D} \leq 290 \mathrm{MeV}$ [3]. The spectrum (29) follows readily from the large distance asymptotics of the heavy-light correlator (12) if we were to use the induced vertices (25) for simplicity. The interaction part in (29) corresponds to the scalar (1) and the (instanton-induced) Coulomb-like-exchange $(\lambda \cdot \lambda)$. The latter is leading and attractive in singlet configurations. Indeed, since the Casimir in the fundamental representation $\left(C_{F}=\lambda \cdot \lambda / 4\right)$ scales with $N_{c}$, the shift in the heavy-light mass is of order $N_{c}^{0}$ with $\alpha_{q Q} \sim N_{c}^{0}$ and $\kappa_{q Q} \sim 1 / N_{c}$

$$
\Delta M_{q Q} \sim-C_{F} \alpha_{q Q} \kappa_{q Q} \sim-\frac{N_{c}}{2} \alpha_{q Q} \kappa_{q Q}
$$


Recall that (26) in the large $N_{c}$ limit gives the following binding energy

$$
\Delta M_{q q} \sim-\left(1-4 S_{q} \cdot S_{q}\right) C_{F} \alpha_{q q} \kappa_{q q}
$$

For spin zero mesons $1-4 S_{q} \cdot S_{q}=4$ so that $\Delta M_{q q} \sim-2 N_{c} \alpha_{q q} \kappa_{q q}$. With this in mind, and using (30), the ratio of the bindings in heavy-light to light-light systems reduces to

$$
\frac{\Delta M_{q Q}}{\Delta M_{q q}} \sim \frac{1}{8}\left(\frac{\Delta M_{Q}}{\Delta M_{q}}\right)\left(\frac{f_{q Q}}{f_{q q}}\right)^{3}
$$

and similarly for the ratio of the bindings for heavy-heavy to light-light systems

$$
\frac{\Delta M_{Q Q}}{\Delta M_{q q}} \sim \frac{1}{8}\left(\frac{\Delta M_{Q}}{\Delta M_{q}}\right)^{2}\left(\frac{f_{Q Q}}{f_{q q}}\right)^{3}
$$

For D-mesons, we have $\Delta M_{D} \sim 0.22 \Delta M_{\pi}\left(f_{D} \sim 290 \mathrm{MeV}\right)$, while for charmonium $\Delta M_{J} \sim 0.10 \Delta M_{\pi}\left(f_{J} \sim 410 \mathrm{MeV}\right)$. The drop in the interaction strength for heavy-heavy systems is compensated by an increase in the decay constant, in comparison to light-light systems. Since the light constituent mass is about $420 \mathrm{MeV}$, the pion binding energy is about $700 \mathrm{MeV}$. Thus the binding energy in heavy-light and heavy-heavy systems is about a $100 \mathrm{MeV}$. These estimates are consistent with the Coulomb estimates in the non-relativistic quark model [13].

Similar relations and estimates hold for baryons. They will be discussed elsewhere along with hyperfine splittings. Subleading effects in $1 / N_{c}$ arise from the left out parts in the color averaging, the non-planar contributions and the perturbative gluon exchanges. They are usually harder to assess without further assumptions.

5. We have explicitly shown how the heavy meson correlator can be analyzed in powers of $1 / m_{Q}$ prior to radiative corrections. Using a random and dilute instanton gas, we have shown that the heavy-light correlator can be calculated in the planar approximation and to leading order in the density fairly accurately. In the random gas the heavy and light 
quarks acquire a mass to order $m_{Q}^{0}$. The induced mass of the heavy quark is related to the perimeter law displayed by the large Wilson loops. The latter reflects on screening in the gas. We have shown that in the long wavelength limit, the interaction induced by instantons between heavy and light quarks is attractive, and both chiral and heavy-quark symmetric. Our construction is immediately amenable to heavy baryons. It also allows for systematic investigations of the $1 / m_{Q}$ corrections and a possible assessment of the $1 / N_{c}$ corrections. Some of these issues will be taken up in the future.

\section{Acknowledgement}

This work has been supported in part by a DOE Grant No. DE-FG02-88ER40388. 


\section{References}

[1] For a review, see e.g., E.V. Shuryak, Phys. Rep. C 115 (1984) 151.

[2] D.I. Diakonov and V.Yu. Petrov, Nucl. Phys. B245, (1984) 259; B272, (1986) 457.

[3] For recent reviews see: M.B. Wise, preprint CALT-68-1860 (1993);

H. Georgi, Preprint HUPT-91-A039 [Published in 1991 TASI Proceedings];

B. Grinstein, Ann. Rev. Nucl. Part. Sci. 42 (1992) 101,

M. Neubert, Preprint SLAC-PUB-6263, 1993

[4] L.L. Foldy and S.A. Wouthuysen, Phys. Rev. 78 (1950) 29.

[5] D.I. Diakonov, V.Yu. Petrov, and P.V. Pobylitsa, Phys. Lett. B 226 (1989) 372.

[6] G.'t Hooft, Phys. Rev. Let 37 (1976) 8, Phys. Rev. D14 (1976) 3432.

[7] J. G. Körner and G. Thompson, Phys. Lett. B 264 (1991) 185; S. Balk, J. G. Körner and D. Pirjol, Mainz Preprint MZ-TH/93-13 (1993), hep-ph/9307230.

[8] M. Luke and A. Manohar, Phys. Lett. B286 (1992) 348.

[9] E. Jenkins and A.V. Manohar, Phys.Lett. B294 (1992) 273.

[10] P.V. Pobylitsa, Phys. Lett. B 226 (1989) 387.

[11] I.I. Bigi, M.A. Shifman, N.G. Uraltsev and A.I. Vainshtein, Preprint TPI-MINN94/4-T, CERN-TH.7171/94, February, 1994; hep-ph/9402360.

S. Narison, CERN-TH.7405/94, August 1994: hep-ph/9408376.

[12] R. Van Royen and V.F. Weisskopf, Nuovo Cim. 50A, (1967) 617.

[13] A. De Rujula, H. Georgi, and S.L. Glashow, Phys. Rev. D 12, (1975) 147. 